\title{
NÍVEL DE ATIVIDADE FÍSICA HABITUAL E DESCONFORTO CORPORAL EM CARTEIROS DE FLORIANÓPOLIS-SC-BRASIL
}

\author{
Level of physical usual activity and body discomfort in postmen of Florianópolis-SC-Brazil
}

\author{
Maria Joanna Cavalheiro1; Adriana Seára Tirloni11,2; Diogo Cunha dos Reis ${ }^{11,3}$; Antônio Renato Pereira Moro1,2,3 \\ 1- Laboratório de Biomecânica - Centro de Desportos - Universidade Federal de Santa Catarina - Florianópolis - SC - \\ Brasil. \\ 2- Programa de Pós-graduação em Engenharia de Produção (Ergonomia) - Centro Tecnológico - Universidade Federal \\ de Santa Catarina - Florianópolis - SC - Brasil. \\ 3- Programa de Pós-graduação em Educação Física - Centro de Desportos - Universidade Federal de Santa Catarina - \\ Florianópolis - SC - Brasil.
}

Resumo: O sedentarismo é um fator de risco para as doenças crônicas não transmissíveis. Objetivo: Analisar o nível de atividade física habitual (NAFH) e desconforto corporal em carteiros lotados nos Centros de Distribuição Domiciliada de Florianópolis em 2008 e verificar a associação entre as variáveis analisadas. Materiais e Métodos: Participaram 105 carteiros que responderam um questionário para verificar o NAFH. Utilizouse o teste Qui-quadrado. Resultados: Constatou-se que 50,5\% dos carteiros tinham excesso de peso e 58,8\% das entregas de correspondências eram a pé. A maioria $(68,9 \%$ ) participava do programa de ginástica na empresa (PGE) e $75,2 \%$ percebiam desconforto corporal. Quanto ao NAFH, $65,7 \%$ eram ativos e $83,3 \%$ tinham lazer ativo. O NAFH teve associação com a faixa etária $(p=0,026)$ e o IMC $(p=0,047)$ e as faixas etárias com o IMC ( $(p=0,001)$ e o meio de transporte $(p=0,002)$. Os carteiros de 31 a 40 anos eram menos ativos fisicamente, com excesso de peso e entregavam as correspondências de forma passiva. Não houve associação da participação no PGE e do desconforto corporal com as variáveis estudadas. Conclusão: A maioria dos carteiros analisados era ativa, classificava suas atividades laborais como moderadas e intensas e tinha lazer ativo.

Palavras-chave: atividade física; trabalho; ginástica.

Abstract: The sedentarism is a factor of risk for the non-transmittable chronic diseases. Objective: Analyze the level of physical usual activity (LPUA) and body discomfort in postman allocateds in Centers for Home Delivery of Florianópolis in 2008 and check the association between variables. Methods and Materials: Participated 105 postmen who has replied to a questionnaire to ascertain the LPUA. The test used Quisquare. Results: It has been noticed that $50.5 \%$ of the postmen were overweight and $58.8 \%$ of the deliveries were made walking. The majority (68.9\%) participates of labor gymnastics program (LGP) and $75.2 \%$ was feeling body discomfort. As the LPUA, 65.7\% were active and $83.3 \%$ had active leisure. The LPUA had association with age $(p=0.026)$ and the BMI $(p=0.047)$ and age with the BMI $(p=0.001)$ and the type of transport $(p=0.002)$. The postmen 31 a 40 years were less physically active, overweight and delivered the mail in a passive way. There was no association of participation in LGP and body discomfort with the variables studied. Conclusion: Most postmen analyzed were active, consider their labor activities moderate and intense and had active leisure.

Keywords: physical activity; workers; gymnastics.

Aceito em: 25/02/2011 - Revista de Educação Física 2012 ABR - 154;21-29. Rio de Janeiro - Brasil

\section{INTRODUÇÃO}

O sedentarismo tem aumentado com a modernização e é um fator de risco para as doenças crônicas não transmissíveis, além de representar um custo econômico à sociedade $^{(1)}$. O processo de industrialização resultou num número crescente de pessoas que se tornaram sedentárias com poucas oportunidades de praticar atividade física(2). Nahas ${ }^{(13)}$ considera um indivíduo sedentário aquele que tem um estilo de vida com o gasto energético inferior a $500 \mathrm{kcal} / \mathrm{semana}$. Blair e Morris ${ }^{(4)}$ citam que o acúmulo de 30 minutos de atividades de intensidade moderada, cinco vezes por semana, pode fornecer importantes benefícios à saúde, e na classificação de Nahas $^{(3)} \mathrm{o}$ indivíduo é dito como moderadamente ativo.
Em contrapartida, Martin et al..$^{(5)}$ preocupam-se quanto a não inclusão de atividades físicas leves na classificação dos níveis de atividades físicas, pois mencionam que essas podem não atender as recomendações de atividade física moderada e vigorosa, porém, citam ser incorreto afirmar que estes tenham um estilo de vida sedentário ou inativo fisicamente.

Outro problema relacionado à industrialização que afeta a saúde é a postura inadequada e os esforços repetitivos realizados ao longo da jornada de trabalho, que poderiam ocasionar desconforto postural e doenças ocupacionais( ${ }^{(6)}$. Bastos Júnior et al. ${ }^{(7)}$ afirmam que a dor é o primeiro sinal de um desconforto corporal que pode até evoluir para uma doença ocupacional, pois o uso 
repetitivo e forçado de grupos musculares e posturas inadequadas pode comprometer a estrutura corporal do indivíduo. A falta de compensação aos esforços do trabaIho pode provocar desconfortos físicos, dores, estresse e absenteísmo(8).

As tarefas realizadas pelos carteiros são variadas, sendo adotadas diferentes posturas: sentados, em pé e em deslocamento. Os carteiros trabalham em Centros de Distribuição Domiciliada (CDD) que são unidades operacionais de onde estes saem para entregar correspondências aos clientes. Nos CDD's é realizada a triagem, que é a separação das correspondências para um determinado destino; a separação por logradouros e a ordenação dos pontos de entrega de cada carteiro utilizando quatro meios de transporte: carro, motocicleta, bicicleta e a pé(9).

Para Almeida et al.(10) a atividade do carteiro é metódica, requer extrema concentração e habilidade manual, além de uma condição razoável de saúde. Os mesmos autores objetivaram identificar os hábitos de vida e gasto calórico despendido nas atividades de distribuição de correspondências utilizando como meio de transporte a bicicleta. Foi constatado que o gasto calórico durante a triagem era de $590 \mathrm{kcal} / \mathrm{dia}$, e na entrega das correspondências aumentava para $1.818 \mathrm{kcal} / \mathrm{dia}$. Apesar do gasto calórico elevado e de proporcionar um bom condicionamento cardiovascular, esta atividade não atende as recomendações do American College of Sport Medicine ${ }^{(11)}$, o qual sugere que os indivíduos desenvolvam quatro componentes da aptidão física relacionada à saúde: composição corporal, aptidão cardiorespiratória, força e resistência muscular e flexibilidade.

Sendo assim, atividades físicas leves e de curta duração que visam diminuir o número de acidentes de trabalho, prevenir doenças ocasionadas por traumas cumulativos e fadiga muscular, corrigir vícios posturais, aumentar a disposição do trabalhador e promover a integração no ambiente de trabalho estão sendo implantadas nas empresas $^{(12) .}$.

Apesar de alguns estudos abordarem o nível de atividade física de trabalhadores, não foi encontrado nenhum que analisasse esta variável em carteiros considerando os diferentes meios de transporte utilizados para a entrega de correspondências e a presença de desconforto corporal. Dessa forma, a presente pesquisa justifica-se pela necessidade dos profissionais que desenvolvem programas de ginástica na empresa (PGE) com carteiros obterem maiores informações sobre o nível de atividade física e desconforto corporal desta população, visando realizarem intervenções adequadas e promoverem a saúde desses trabalhadores. Diante do exposto, o objetivo deste estudo foi analisar o nível de atividade física habitual (NAFH) e o desconforto corporal em carteiros de Florianópolis e verificar a associação entre as variáveis: sexo, faixa etária, grau de escolaridade, Índice de Massa Corporal (IMC), NAFH, meio de transporte utilizado na entrega das correspondências, participação no PGE e desconforto corporal.

\section{MATERIAIS E MÉTODOS}

Este estudo caracterizou-se como uma pesquisa de corte transversal com abordagem quantitativa, na qual participaram 105 carteiros, 84 homens $(33,9 \square 8,3$ anos de idade) e 21 mulheres (33,2 $\square 7,3$ anos de idade), sendo que para a análise dos dados a idade foi categorizada em faixas etárias: 18-30 anos; de 31-40 anos e > 40 anos. Os trabalhadores estavam lotados nos cinco Centros de Distribuição Domiciliada (CDD) da Empresa Brasileira de Correios e Telégrafos da cidade de Florianópolis-SC. A atividade laboral dos carteiros consistia na distribuição de correspondências em escaninhos durante a manhã (triagem), onde realizavam movimentos repetitivos com as mãos e estes permaneciam ora na posição em pé ora na posição sentada. Já no período vespertino, realizavam a entrega domiciliar utilizando um dos meios de transporte disponibilizados pela empresa: carro, motocicleta, bicicleta e a pé. Todos os trabalhadores tinham o mesmo vínculo empregatício, haja vista que o processo de admissão dos trabalhadores era realizado por meio de concurso público, sendo o nível de escolaridade exigido no ano da coleta o ensino médio.

Primeiramente foi realizado o contato com os responsáveis pelo programa de ginástica na empresa (PGE) dos Correios, os quais receberam explicações sobre a relevância, os objetivos e procedimentos para a realização desta pesquisa. Após o consentimento dos responsáveis, este projeto de pesquisa foi submetido e aprovado pelo Comitê de Ética em Pesquisa com Seres Humanos da Universidade Estadual de Santa Catarina, processo $n^{\circ} 152 / 2007$.

Conforme informações repassadas pelos gerentes dos CDD's existiam 210 carteiros lotados nos cinco CDD's de Florianópolis. Os critérios de inclusão dos participantes foram: não estar afastado da empresa, aceitar participar 
da pesquisa voluntariamente e responder o questionário. Desta forma, 132 trabalhadores concordaram em participar da pesquisa, sendo que 27 foram excluídos, pois não responderam algumas perguntas do questionário.

No PGE eram realizados exercícios de alongamento, postura e consciência corporal em sessões de 10 minutos no próprio setor de trabalho. Além disso, os profissionais do PGE orientavam os trabalhadores a praticarem atividade física e se alimentarem corretamente, para meIhorar a qualidade de vida e a produtividade.

Como instrumento de medida, foi utilizado um questionário contendo perguntas fechadas sobre características pessoais (sexo, idade, escolaridade, massa corporal, estatura e nível de atividade física habitual (NAFH)) e profissionais (meio de transporte utilizado na entrega das correspondências; participação e frequência semanal no PGE) dos trabalhadores.

Para a análise do NAFH foi utilizado um questionário desenvolvido por Pate ${ }^{(13)}$ e adaptado por Nahas $^{(3)}$ que contêm questões referentes às atividades ocupacionais e atividades de lazer. Cada questão assinalada corresponde a um valor e a soma desses valores classifica o NAFH (0-5: inativo; 6-11: moderadamente ativo; 12-20: ativo; $\geq 21$ : muito ativo). Segundo os autores a faixa ideal para a maioria das pessoas é de 12-20 pontos. A escolha desse instrumento se deu devido a esse ser recomendado para adultos jovens (20 a 39 anos) ${ }^{(3)}$, faixa etária prevalente entre carteiros $(10,14,15,16)$ e também, por não ser um questionário recordatório, pois segundo Hallal et al.(17), as pessoas apresentam dificuldade para lembrar de detalhes referentes às suas atividades físicas ao responderem questionários recordatórios.

Para verificar a presença de desconfortos corporais foi utilizada uma figura, desenvolvida por Polito e Bergamaschi(18) adaptada para este estudo, na qual o trabaIhador, caso sentisse dor, assinalaria a região do corpo (pescoço, cotovelo, antebraço, punho/mão, coxa, perna, ombro, costas, coluna lombar, quadril, joelhos e tornozelo/pé) e a frequência (ocasionalmente ou frequentemente) que a sentia.

A coleta de dados ocorreu nos CDD's, pelo orientador de atividade física de cada CDD, devidamente treinado para a aplicação do questionário. Todos os trabalhadores foram convidados a participar do estudo, comunicados sobre os procedimentos de pesquisa, informados sobre a relevância da realização do mesmo e a garantia do sigilo de identificação dos participantes. Os trabalhadores que aceitaram participar assinaram o Termo de Consentimento Livre e Esclarecido. Para a aplicação do questionário foi utilizado o horário do PGE, pois neste período todos os carteiros poderiam parar de trabalhar.

Os dados foram organizados no software SPSS 15.0 Windows ${ }^{\circledR}$ (SPSS Inc., Chicago IL, EUA). Utilizou-se a estatística descritiva mediante frequência simples e percentual. Para verificar a associação entre as variáveis categóricas (sexo, faixa etária, grau de escolaridade, IMC, NAFH, meio de transporte utilizado na entrega das correspondências, participação no PGE e desconforto corporal) foi utilizado o teste Qui-quadrado, no qual foi adotado nível de significância de $5 \%$.

\section{RESULTADOS}

A descrição das características dos carteiros pesquisados é apresentada na TABELA 1, demonstrando as frequências e percentuais em relação à faixa etária, grau de escolaridade, IMC, NAFH, meio de transporte utilizado para a entrega das correspondências, participação no PGE e presença de desconforto corporal.

TABELA1- CARACTERÍSTICAS DOS CARTEIROS QUANTO À FAIXA ETÁRIA, GRAU DE ESCOLARIDADE, ÍNDICE DE MASSA CORPORAL (IMC), NÍVEL DE ATIVIDADE FÍSICA HABITUAL (NAFH), MEIO DE TRANSPORTE PARAA ENTREGA DAS CORRESPONDÊNCIAS, PARTICIPAÇÃO NO PGE E PRESENÇA DE DESCONFORTO CORPORAL.

\begin{tabular}{|c|c|c|c|}
\hline \multicolumn{2}{|l|}{ Faixa etária } & $\mathbf{n}$ & $\%$ \\
\hline \multicolumn{2}{|l|}{18 a 30 anos } & 41 & 39,0 \\
\hline \multicolumn{2}{|l|}{31 a 40 anos } & 42 & 40,0 \\
\hline \multicolumn{2}{|l|}{$>40$ anos } & 22 & 21,0 \\
\hline \multicolumn{4}{|c|}{ Grau de escolaridade } \\
\hline \multicolumn{2}{|l|}{ Fundamental } & 3 & 2,9 \\
\hline \multicolumn{2}{|l|}{ Médio } & 74 & 71,9 \\
\hline \multicolumn{2}{|l|}{ Superior } & 26 & 25,2 \\
\hline \multicolumn{4}{|c|}{ Índice de Massa Corporal - IMC* (Kg/m2) } \\
\hline \multirow[t]{2}{*}{$\begin{array}{l}\text { Baixo peso/ } \\
\text { Normal }\end{array}$} & $\begin{array}{l}\text { Baixo peso } \\
\text { - Abaixo de } \\
18,5\end{array}$ & 2 & 1,9 \\
\hline & $\begin{array}{l}\text { Normal - } 18,5 \\
\text { a } 24,9\end{array}$ & 50 & 47,6 \\
\hline
\end{tabular}




\begin{tabular}{|c|c|c|c|}
\hline \multirow[t]{2}{*}{$\begin{array}{l}\text { Excesso de } \\
\text { peso }\end{array}$} & $\begin{array}{l}\text { Sobrepeso - } \\
25 \text { a } 29,9\end{array}$ & 47 & 44,8 \\
\hline & $\begin{array}{l}\text { Obesidade - } \\
>30\end{array}$ & 6 & 5,7 \\
\hline \multicolumn{4}{|l|}{ NAFH } \\
\hline \multirow{2}{*}{$\begin{array}{l}\text { Insuficien- } \\
\text { temente } \\
\text { ativos }\end{array}$} & Inativos & 8 & 7,6 \\
\hline & $\begin{array}{l}\text { Moderadamente } \\
\text { ativos }\end{array}$ & 28 & 26,7 \\
\hline \multirow[t]{2}{*}{ Ativos } & Ativos & 45 & 42,9 \\
\hline & Muito ativos & 24 & 22,8 \\
\hline \multicolumn{4}{|c|}{ Meio de transporte para a entrega de correspondências } \\
\hline \multirow[t]{2}{*}{ Passivo } & Carro & 2 & 2,0 \\
\hline & Moto & 35 & 36,1 \\
\hline \multirow[t]{2}{*}{ Ativo } & Bicicleta & 3 & 3,1 \\
\hline & A pé & 57 & 58,8 \\
\hline \multicolumn{4}{|c|}{ Participação no PGE } \\
\hline \multicolumn{2}{|l|}{ Não participa } & 32 & 31,1 \\
\hline \multicolumn{2}{|l|}{ Participa } & 71 & 68,9 \\
\hline \multicolumn{4}{|l|}{ Desconforto } \\
\hline \multicolumn{2}{|l|}{ Ausente } & 26 & 24,8 \\
\hline \multicolumn{2}{|l|}{ Presente } & 79 & 75,2 \\
\hline \multicolumn{4}{|c|}{$\begin{array}{l}\text { *Classificação conforme recomendações da Organização Mundial } \\
\text { da Saúde(29) } \\
\text { PGE - Programa de Ginástica na Empresa }\end{array}$} \\
\hline
\end{tabular}

Conforme a TABELA 1 pode-se constatar que a maioria dos trabalhadores estava nas faixas etárias de 18-30 anos e de 31-40 anos (39 e 40\%, respectivamente) e tinha nível médio de escolaridade $(71,9 \%)$.

O estado nutricional dos trabalhadores foi de $24 \pm 3,2 \mathrm{~kg} /$ m2 e observou-se que $47,6 \%$ foram classificados com IMC normal e 44,8\% com sobrepeso, havendo poucos com baixo peso e obesidade. Os dados foram agrupados em categorias dicotômicas para análise estatística, sendo classificado como baixo peso/ normal quem apresentou IMC $\leq 24,9 \mathrm{Kg} / \mathrm{m} 2$ e excesso de peso quem apresentou IMC $\geq 25 \mathrm{Kg} / \mathrm{m} 2$, assim, $50,5 \%$ dos carteiros foram classificados com excesso de peso.

Quanto ao NAFH, 22,8\% foram considerados muito ativos, $42,9 \%$ ativos, $26,7 \%$ moderadamente ativos e $7,6 \%$ inativos. Os dados foram agrupados em categorias dicotômicas para análise estatística, no qual foi classificado como insuficientemente ativo quem obteve pontuação de $0-11$ pontos e ativo $\geq 12$ pontos, desta maneira, $65,7 \%$ dos carteiros foram classificados como ativos.

Quando questionados sobre como as suas atividades laborais poderiam ser descritas, $92,5 \%$ citaram que na maior parte do dia realizavam atividades moderadas a intensas. Sobre as atividades de lazer, 83,3\% faziam atividade física durante o lazer, $52,1 \%$ dos carteiros mencionaram realizar exercícios aeróbios, 3,1\% exercícios de resistência muscular e 28,1\% realizavam

tanto exercícios aeróbios como de resistência muscular, sendo que $35,4 \%$ relataram que realizavam exercícios físicos para relaxar.

Verificou-se que mais da metade dos carteiros entregava as correspondências de forma ativa (bicicleta/a pé) $(61,9 \%)$ e participava do PGE $(68,9 \%)$, sendo que destes $71,8 \%$ faziam ginástica na empresa de três a cinco vezes por semana.

Foi constatado que $75,2 \%$ dos carteiros sentiam desconforto, sendo que estes ocorriam principalmente nas costas $(50,5 \%)$, lombar $(45,7 \%)$, ombro $(44,8 \%)$, pescoço $(42,8 \%)$ e joelhos $(41,9 \%)$ (TABELA 2$)$.

TABELA 2 - PRESENÇA DE DESCONFORTO POR REGIÕES CORPORAIS EM CARTEIROS DE FLORIANÓPOLIS-SC.

\begin{tabular}{|l|l|l|l|l|}
\hline Desconforto Corporal \\
\hline Regiões & \multirow{2}{*}{$\begin{array}{l}\text { Sem dor } \\
\text { (\%) }\end{array}$} & \multicolumn{4}{|l|}{ Com dor $(\%)$} & \multicolumn{2}{l|}{} \\
\cline { 3 - 5 } & & $\begin{array}{l}\text { Ocasio- } \\
\text { nalmente }\end{array}$ & $\begin{array}{l}\text { Frequen- } \\
\text { temente }\end{array}$ & Total \\
\hline Pescoço & 57,2 & 29,5 & 13,3 & 42,8 \\
\hline Cotovelo & 80,0 & 15,2 & 4,8 & 20,0 \\
\hline Antebraço & 77,1 & 14,3 & 8,6 & 22,9 \\
\hline Punho & 60,0 & 22,9 & 17,1 & 40,0 \\
\hline Coxa & 82,9 & 12,4 & 4,8 & 17,2 \\
\hline Perna & 69,5 & 17,2 & 13,3 & 30,5 \\
\hline Ombro & 55,2 & 20,0 & 24,8 & 44,8 \\
\hline Costas & 49,5 & 25,7 & 24,8 & 50,5 \\
\hline Lombar & 54,3 & 19,0 & 26,7 & 45,7 \\
\hline Quadril & 83,8 & 8,6 & 7,6 & 16,2 \\
\hline Joelho & 58,1 & 26,7 & 15,2 & 41,9 \\
\hline Tornozelo & 68,6 & 19,0 & 12,4 & 31,4 \\
\hline
\end{tabular}

Na TABELA 3 estão apresentadas as associações entre as faixas etárias com o IMC, meio de transporte utilizado para entrega de correspondências, participação no PGE e desconforto corporal.

\begin{tabular}{|c|c|c|c|c|c|c|c|}
\hline \multicolumn{2}{|l|}{ Características } & \multicolumn{6}{|c|}{ Faixas etárias } \\
\hline & & \multirow{2}{*}{$\begin{array}{l}18 \text { a } \\
30 \\
\text { anos } \\
\%\end{array}$} & \multirow{2}{*}{$\begin{array}{l}31 \mathrm{a} \\
40 \\
\text { anos } \\
\mathrm{n}\end{array}$} & \multirow{2}{*}{$\begin{array}{l}>40 \\
\text { anos }\end{array}$} & \multirow[b]{2}{*}{$n$} & \multirow[b]{2}{*}{$\%$} & \multirow[b]{2}{*}{$p$} \\
\hline Sexo & $\mathrm{n}$ & & & & & & \\
\hline Feminino & 09 & 42,9 & 07 & 33,3 & 05 & 23,8 & $\dagger$ \\
\hline Masculino & 32 & 38,1 & 35 & 41,7 & 17 & 20,2 & \\
\hline \multicolumn{8}{|c|}{ Grau de escolaridade } \\
\hline Ensino médio & 27 & 36,5 & 32 & 43,2 & 15 & 20,3 & \multirow[t]{2}{*}{$\dagger$} \\
\hline $\begin{array}{l}\text { Ensino } \\
\text { Superior }\end{array}$ & 14 & 53,8 & 10 & 38,5 & 02 & 7,7 & \\
\hline
\end{tabular}




\begin{tabular}{|c|c|c|c|c|c|c|c|}
\hline Normal & 29 & 55,8 & 12 & 23,1 & 11 & 21,2 & \multirow[t]{2}{*}{$0,001^{*}$} \\
\hline Sobrepeso & 12 & 22,6 & 30 & 56,6 & 11 & 20,8 & \\
\hline \multicolumn{8}{|c|}{ Meio de transporte para a entrega de correspondências } \\
\hline $\begin{array}{l}\text { Passivo } \\
\text { (carro/moto) }\end{array}$ & 10 & 27,0 & 24 & 64,9 & 3 & 8,1 & \multirow[t]{2}{*}{$0,002^{*}$} \\
\hline $\begin{array}{l}\text { Ativo } \\
\text { (bicicleta/a } \\
\text { pé) }\end{array}$ & 29 & 48,3 & 17 & 28,3 & 14 & 23,3 & \\
\hline \multicolumn{8}{|c|}{ Participação no PGE } \\
\hline Não Participa & 12 & 37,5 & 12 & 37,5 & 8 & 25,0 & \multirow[t]{2}{*}{0,831} \\
\hline Participa & 29 & 40,8 & 28 & 39,4 & 14 & 19,7 & \\
\hline \multicolumn{8}{|c|}{ Desconforto corporal } \\
\hline Ausente & 8 & 30,8 & 12 & 46,2 & 6 & 23,1 & \multirow[t]{2}{*}{0,604} \\
\hline Presente & 33 & 41,8 & 30 & 38,0 & 16 & 20,3 & \\
\hline
\end{tabular}

Observou-se associação entre faixas etárias com IMC $(p=0,001)$ e os meios de transporte utilizados para a entrega de correspondências $(p=0,002)$, na qual a maioria dos carteiros que apresentou excesso de peso estava na faixa etária de 31-40 anos.

As associações entre o NAFH e as variáveis: sexo, faixa etária, grau de escolaridade, IMC, meio de transporte utilizado para a entrega de correspondências, participação no PGE e desconforto corporal estão apresentadas na TABELA 4.

TABELA 4 - ASSOCIAÇÃO ENTRE NÍVEL DE ATIVIDADE FÍSICA HABITUAL (NAFH) COM SEXO, FAIXAETÁRIA, GRAUDEESCOLARIDADE, ÍNDICEDE MASSA CORPORAL (IMC), MEIO DE TRANSPORTE PARA A ENTREGA DE CORRESPONDÊNCIAS, PARTICIPAÇÃO NO PGE E DESCONFORTO CORPORAL.

\begin{tabular}{|c|c|c|c|c|c|}
\hline \multirow[t]{2}{*}{ Características } & \multicolumn{4}{|c|}{ NAFH } & \multirow[b]{3}{*}{$\mathrm{p}$} \\
\hline & \multicolumn{2}{|c|}{$\begin{array}{l}\text { Insuficientemente } \\
\text { ativos }\end{array}$} & \multicolumn{2}{|c|}{ Ativos } & \\
\hline Sexo & $\mathbf{n}$ & $\%$ & $\mathbf{n}$ & $\%$ & \\
\hline Feminino & 9 & 42,9 & 12 & 57,1 & \multirow[t]{2}{*}{0,355} \\
\hline Masculino & 27 & 32,1 & 57 & 67,9 & \\
\hline \multicolumn{6}{|l|}{ Faixa etária } \\
\hline 18 a 30 anos & 8 & 19,5 & 33 & 80,5 & $0,026^{*}$ \\
\hline 31 a 40 anos & 20 & 47,6 & 22 & 52,4 & \\
\hline$>$ que 40 anos & 8 & 36,4 & 14 & 63,6 & \\
\hline \multicolumn{6}{|c|}{ Grau de escolaridade } \\
\hline Ensino médio & 26 & 35,1 & 48 & 64,9 & \multirow[t]{2}{*}{0,686} \\
\hline Ensino Superior & 8 & 30,8 & 18 & 69,2 & \\
\hline IMC & & & & & \\
\hline
\end{tabular}

\begin{tabular}{|c|c|c|c|c|c|}
\hline Normal & 13 & 25 & 39 & 75 & \multirow[t]{2}{*}{$0,047^{*}$} \\
\hline Excesso de peso & 23 & 43,4 & 30 & 56,6 & \\
\hline \multicolumn{6}{|c|}{ Meio de transporte para a entrega de correspondências } \\
\hline $\begin{array}{l}\text { Passivo (carro/ } \\
\text { moto) }\end{array}$ & 15 & 40,5 & 22 & 59,5 & \multirow[t]{2}{*}{0,155} \\
\hline Ativo (bicicleta/a pé) & 16 & 26,7 & 44 & 73,3 & \\
\hline \multicolumn{6}{|l|}{ Participação no PGE } \\
\hline Não Participa & 10 & 31,3 & 22 & 68,7 & \multirow[t]{2}{*}{0,694} \\
\hline Participa & 25 & 35,2 & 46 & 64,8 & \\
\hline \multicolumn{6}{|l|}{ Desconforto corporal } \\
\hline Ausente & 11 & 42,3 & 15 & 57,7 & \multirow[t]{2}{*}{0,320} \\
\hline Presente & 25 & 31,6 & 54 & 68,4 & \\
\hline
\end{tabular}

Ao verificar o nível de associação do NAFH com as variáveis categóricas constatou-se que houve associação entre as faixas etárias $(p=0,026)$, sendo que os carteiros de 18-30 anos foram os mais ativos comparados com os carteiros das outras faixas etárias. Também verificou-se associação entre o NAFH e o IMC $(p=0,047)$, pois $75 \%$ dos indivíduos que apresentaram IMC classificados como baixo peso/normal eram ativos fisicamente.

Não foi constatada associação da participação no PGE com as variáveis: sexo $(p=0,182)$, IMC $(p=0,432)$, meio de transporte para a entrega de correspondências $(p=0,455)$ e presença de desconforto $(p=0,908)$, porém observou-se que dos que sentiam dor, $30,8 \%$ não participavam do programa e $69,2 \%$ participavam.

Quanto ao desconforto corporal, este não apresentou associação com as variáveis sexo $(p=0,214)$, IMC $(p=0,955), \operatorname{NAFH}(p=0,320)$ e meio de transporte para a entrega de correspondências $(p=0,682)$. $O I M C$ não teve associação com as variáveis: sexo $(p=0,435), P G E$ $(p=0,432)$ e com o meio de transporte $(p=0,480)$.

\section{DISCUSSÃO}

A média de idade observada no presente estudo $(33,7$ anos) foi muito próxima às observadas em outros estudos com carteiros (31,3 e 31,6 anos, respectivamente) ${ }^{(10,15,16)}$.

Constatou-se associação entre as faixas etárias e o NAFH ( $p=0,026)$, o IMC $(p=0,001)$ e o meio de transporte para a entrega de correspondências $(p=0,002)$. Dessa forma, verificou-se que os carteiros que estavam na 
nas faixas etárias 18-30 anos e > 40 anos eram mais ativos fisicamente que os da faixa 31-40 anos. Nessa mesma faixa etária foram observados mais carteiros com excesso de peso $(56,6 \%)$ e os meios de transporte mais utilizados para a entrega de correspondências eram os passivos (carro/moto) $(64,9 \%)$ (TABELA 3 ), mostrando a importante relação do nível de atividade física com o IMC e o meio de transporte nessa faixa etária.

Quanto ao grau de escolaridade, observou-se no presente estudo que $71,9 \%$ dos carteiros concluíram o ensino médio e $25,2 \%$ o ensino superior, diferente do estudo de Almeida et al. ${ }^{(10)}$, no qual, $73,9 \%$ dos carteiros concluíram o ensino médio e apenas $4,4 \%$ o ensino superior. Gomes, Siqueira e Sichieri(19) realizaram um estudo com o objetivo de descrever três marcadores de atividade física (ocupacional, lazer e horas assistindo televisão) em moradores do Rio de Janeiro. Utilizaram como instrumento o Questionário Internacional de Atividade Física (IPAQ) e observaram que quanto maior o grau de escolaridade, maior era a frequência de atividades físicas de lazer em ambos os sexos e menos horas eram despendidas assistindo televisão entre as mulheres. Diferentemente do presente estudo que não houve associação entre o grau de escolaridade e o NAFH $(p=0,686)$, talvez devido à própria atividade laboral exigir uma boa condição física e mental, e o grau de escolaridade mínimo para o ingresso nesta profissão ser o ensino médio(20). Apesar disso, dois carteiros do presente estudo (com 49 e 50 anos de idade) tinham apenas o ensino fundamental, pois quando ingressaram na empresa a exigência de escolaridade era menor.

Gomes, Siqueira e Sichieri(19) afirmaram que um dos poucos fatores que podem prevenir o ganho de massa corporal é a prática regular de exercícios físicos. Foi o que indicou o estudo atual, pois houve associação entre o IMC e o NAFH, sendo que os carteiros com IMC baixo peso/normal mostraram-se significativamente mais ativos $(p=0,047)$. Contudo, a maioria $(50,5 \%)$ dos carteiros estava com sobrepeso $(44,8 \%)$ ou obesidade $(5,7 \%)$, apesar da atividade laboral de distribuição de correspondências apresentar gasto calórico de moderado a intenso(16). Esse fato pode estar relacionado com a forma de entrega das correspondências, pois na forma ativa o percentual de trabalhadores com excesso de peso foi menor.

Resultados similares aos supracitados também foram constatados por Almeida et al.(10) que tinham como objetivo identificar hábitos de vida e gasto calórico despendido nas atividades de distribuição de correspondências de 37 carteiros que utilizavam a bicicleta para as entregas. Utilizando o questionário IPAQ, observaram que $73,9 \%$ dos carteiros participavam de atividade vigorosa por prazer ou com objetivos esportivos, sendo que $34,7 \%$ desses praticavam duas vezes por semana. Quanto ao gasto calórico despendido nos dois períodos de trabalho (triagem e entrega de correspondências), somou-se $1818 \mathrm{kcal} / \mathrm{dia}$. Pagani e Duarte ${ }^{(16)}$ analisaram o carteiro convencional $(n=28)$ a pé e verificaram a relação entre as atividades realizadas durante o trabalho e a saúde. O nível de aptidão física geral do grupo foi moderado, o gasto energético no trabalho foi em média de $1.769 \mathrm{kcal}$, caracterizando como uma atividade laboral de moderada a pesada. Verificou-se que os próprios carteiros do atual estudo $(92,5 \%)$ classificaram subjetivamente as suas atividades laborais como moderadas a intensas na maior parte do dia, apesar do NAFH não estar associada com o meio de transporte utilizado para a entrega de correspondências $(p=0,155)$.

No estudo de Sena et al.(21) foi constatado diferença significativa entre as variáveis antropométricas (massa corporal, estatura, IMC, relação cintura quadril e percentual de gordura) de carteiros $(n=53)$ e taxistas $(n=52)$, sendo que todos os carteiros eram ativos fisicamente e tinham composição corporal satisfatória, já os taxistas, que realizavam suas atividades laborais sentados, a maioria $(55,8 \%)$ era sedentária. O mesmo ocorreu no presente estudo, pois os carteiros que entregavam as correspondências de forma passiva (carro/moto) apresentaram IMC maiores.

Com o objetivo de avaliar o nível de atividade física da população do estado de São Paulo, Matsudo et al.(1) classificaram os sujeitos investigados em muito ativo, ativo, irregularmente ativo e sedentário, utilizando como instrumento o IPAQ. Os resultados demonstraram que $42,5 \%$ dos homens e $48,6 \%$ das mulheres eram ativos, no presente estudo, apesar de utilizar outro instrumento, constatou-se que $65,7 \%$ dos carteiros eram ativos, e $33,3 \%$ inativos, sendo que $67,9 \%$ das mulheres e $57,1 \%$ dos homens eram ativos, não havendo associação entre o NAFH e o sexo $(p=0,355)$, o que pode ser justificado pela exigência profissional para exercer o cargo de agente de correios, não existindo distinção entre os sexos quanto ao condicionamento físico.

Barros e Nahas ${ }^{(22)}$ também utilizaram o questionário IPAQ para identificar a prevalência e analisar a associação entre comportamentos de risco à saúde, percepção de estresse e autoavaliação do nível de saúde em 4.225 trabalhadores da indústria. Desses, $46,2 \%$ não realizavam atividades físicas no lazer, sendo que $67 \%$ eram mulheres e $34,8 \%$ homens. A prevalência de sujeitos insuficientemente ativos no lazer foi maior entre as mulheres e aumentou proporcionalmente com a idade, independente do sexo.

No estudo de Gomes, Siqueira e Sichieri(19) $18,4 \%$ dos homens e $9,1 \%$ das mulheres realizavam 
frequentemente alguma atividade física de lazer regular ou esporte, já $59,8 \%$ dos homens e $77,8 \%$ das mulheres referiram nunca ter praticado atividade física de lazer. Observou-se que as mulheres praticavam atividades de lazer de menor duração e gasto energético que os homens. Também verificou-se que homens e mulheres com atividade laboral moderada e pesada tenderam a fazer menos atividade física de lazer do que os sem atividade ou com atividade laboral leve. Contrapondo, no presente estudo $92,5 \%$ dos carteiros perceberam as atividades laborais como moderadas a intensas e $83,3 \%$ desses realizavam atividades físicas no momento de lazer.

Baretta, Baretta e Peres ${ }^{(23)}$ realizaram um estudo que objetivou estimar o nível de atividade física da população de Joaçaba-SC, utilizaram o IPAQ como instrumento, e observaram que a prevalência de inatividade física foi de $57,4 \%$. Por meio de entrevistas, Pitanga e Lessa(2) verificaram a prevalência e determinantes do sedentarismo no lazer em adultos na cidade de Salvador-BA, e constataram que $72,5 \%$ dos moradores eram sedentários no lazer. No atual estudo apenas $7,6 \%$ dos sujeitos analisados eram inativos, sendo um percentual baixo quando comparado aos estudos citados anteriormente.

Ao compararem o nível de atividade física de 323 trabalhadores da área administrativa de uma empresa de acordo com a participação em PGE, por meio do IPAQ, Lima, Romero e Ceschini ${ }^{(24)}$ comprovaram que os trabalhadores participantes do PGE eram mais ativos fisicamente quando comparados com os trabalhadores que não participavam do programa. No presente estudo não houve associação significativa entre o NAFH e a participação no PGE $(p=0,694)$, pois $68,7 \%$ dos trabalhadores que não participavam e $64,8 \%$ dos que participavam eram ativos.

No estudo de Almeida et al. ${ }^{(10)}, 78 \%$ dos carteiros relataram estar em boas condições de saúde, no entanto, o principal problema citado foi dor na coluna, com $26 \%$ de ocorrência. Pagani e Duarte ${ }^{(15,16)}$ realizaram avaliações de aptidão física e postural durante o trabalho, e aplicaram um questionário estruturado para caracterizar o grupo e obter informações referentes à saúde no trabalho. Observou-se que o índice de dispensa médica foi de $50 \%$, nas quais os Distúrbios Osteomusculares Relacionados ao Trabalho (DORT) foram o principal motivo (56\%), e a principal doença foi a lombalgia (36,5\%). Mesmo os carteiros exercendo atividades variadas durante a jornada de trabalho (triagem e entrega de correspondências), verificou-se no presente estudo que $50,5 \%$ dos carteiros sentiam desconforto nas costas e $45,7 \%$ na região lombar, além dos ombros $(44,8 \%)$, pescoço $(42,8 \%)$ e joelhos $(41,9 \%)$, regiões essas, que devem ser levadas em consideração no planejamento da ginástica laboral.

Wahlstedt(14) realizou um estudo em Estocolmo com 655 carteiros com o objetivo de analisar a possível associação entre fatores psicossociais, condições do trabalho e reclamações musculoesqueléticas. Utilizouse um questionário referente a exigências psicológicas do trabalho, contato com supervisores e companheiros de equipe, qualidade de sono, tempo de serviço, carga física e reclamações musculoesqueléticas. Os carteiros relataram alta exigência psicológica e baixa carga física de trabalho, além de reclamações de desconfortos no pescoço e na coluna lombar, sendo que aqueles com baixa habilidade de trabalho e mais tempo de serviço apresentaram mais dores lombares. Carteiros com alta carga física e alta exigência psicológica tiveram reclamações lombares e não no pescoço, diferentemente do presente estudo em que ambas as regiões foram citadas.

Romero, Lima e Ceschini ${ }^{(25)}$ compararam a prevalência de dores corporais de acordo com a participação em PGE em 440 trabalhadores de ambos os sexos da área administrativa de uma empresa, para tal objetivo foi utilizado um questionário para identificar as dores corporais. Os autores concluíram que essa prevalência foi de $38,2 \%$, sendo mais elevada em homens $(44,6 \%)$ do que em mulheres $(36,8 \%)$. Em relação ao grupo que não participou do $P G E$, os resultados demonstraram uma chance de 2,95 vezes maior dos funcionários apresentarem dores corporais quando comparados aos funcionários que participaram do programa. No presente estudo foi verificada a presença de desconfortos corporais em ambos os grupos, tanto nos que não participavam do PGE $(75 \%)$ como nos que participavam (76\%), não havendo diferença significativa entre os grupos $(p=0,908)$ bem como entre os sexos $(p=0,214)$. Salientando que $69,2 \%$ dos trabalhadores que sentiam desconforto participavam do programa, segundo Sjögaard et al. ${ }^{(26)}$ estudos têm revelado que a participação em PGE tem efeito positivo no alívio da dor, talvez por esse motivo a maioria dos carteiros que sentiam desconforto corporal participava do PGE, no entanto não foi constatada associação entre 0 NAFH e o desconforto $(p=0,320)$. Como o DORT não possui uma causa única e determinada ${ }^{(28)}$ e apesar da eficácia da ginástica laboral na sua prevenção não ser comprovada (27), a mesma vem atuando como uma ferramenta para promover a saúde dos trabalhadores, conscientizá-los sobre postura e auxiliar a reduzir o desconforto corporal, sendo primordial nas empresas a realização de ações ergonômicas que melhorem as condições de trabalho e diminuam a incidência de DORT. 


\section{CONCLUSÕES}

Diante dos resultados deste estudo conclui-se que a maioria dos carteiros pesquisados era do sexo masculino, tinha o ensino médio era ativa fisicamente, tinha lazer ativo e sentia desconforto corporal, sendo mais acometidos nas regiões das costas, lombar, ombro, pescoço e joelhos, regiões essas que devem ser levadas em consideração no planejamento dos exercícios da ginástica na empresa.

Ao testar a associação entre as variáveis, constatouse que houve associação do NAFH com a faixa etária e o IMC; e também, entre a faixa etária e o IMC e o meio de transporte para a entrega de correspondências, mas não houve associação entre a participação no PGE e o desconforto corporal com as variáveis pesquisadas. Dessa forma, salienta-se a importância da orientação de carteiros, principalmente aqueles que entregam as correspondências de forma passiva, sobre os riscos relacionados à saúde, como o estado nutricional e o sedentarismo.

\section{Agradecimentos:}

Ao CNPq e a CAPES-REUNI pelas bolsas do segundo e terceiro autores.

\section{REFERÊNCIAS}

1. Matsudo SM, Matsudo VR, Araujo T, Andrade D, Andrade E, Oliveira L, et al. Nível de atividade física da população do Estado de São Paulo: análise de acordo com o gênero, idade, nível socioeconômico, distribuição geográfica e de conhecimento. Rev Bras Ciênc Mov 2002;10:41-50.

2. Pitanga FJG, Lessa I. Prevalência e fatores associados ao sedentarismo no lazer em adultos. Cad Saúde Púb 2005;21:870-7.

3. Nahas MV. Atividade física, saúde e qualidade de vida: conceitos e sugestões para um estilo de vida ativo. 5. ed. Londrina: Midiograf; 2010.

4. Blair SN, Morris JN. Healthy hearts-and the universal benefits of being physically active: physical activity and health. Annals Epidem 2009;19:253-6.

5. Martin L, Garcia T, Silva KS, Nahas MV. Atividades físicas e comportamentos sedentários: evidências e novos caminhos para nossas pesquisas. Rev Bras Ativ Fís Saúde 2010;15:126-7.

6. Silva J, Taranto IC, Piaseck F. Ginástica laboral: alongamento x flexionamento. SaBios - Revista Saúde e Biologia 2006;1:6-12.

7. Bastos Júnior LAD, Silva GBS, Vieira HANF. Avaliação da prevalência de síndrome do piriforme em motoristas de ônibus, no município de Gov. ValadaresMG. Disponível em: http://www.wgate.com.br/conteudo/ medicinaesaude/ fisioterapia/traumato/piriforme_luiz/ piriforme_luiz.htm (07 jan. 2011).

8. Salve MGC, Theodoro PFR. Saúde do trabalhador: a relação entre ergonomia, atividade física e qualidade de vida. Salusvita 2004;23:137-46.

9. Araldi AAR. Localização de centro de distribuição de correios numa cidade de médio porte. Rev Prod 1997;6:139-49.

10. Almeida EB, Xavier GNA, Carminatti LJ, Della Giustina MC. Gasto calórico nas atividades de trabalho e cotidianas, dos carteiros que utilizam bicicleta. Rev Bras Cineantropom Desempenho Hum 2004;6:53-61.

11. American College of Sport Medicine. ACSM's Guidelines for exercise testing and prescription. Philadelphia: Lippincott Williams \& Wilkins; 2006.

12. Martins CO, Duarte MFS. Efeitos da Ginástica Laboral em servidores da Reitoria da UFSC. Rev Bras Ciênc Mov 2000;8:07-13.

13. Pate RR, Pratt M, Blair SN, Haskel WL, Macera CA, Bouchard C, et al. Physical activity and public health: A recommendation from the Centers for Disease Control and Prevention and the American College of Sports Medicine. JAMA 1995;273:402-407.

14. Wahlstedt KGI. Postal work: work organizational changes as tools to improve health. 2001. 88f. Dissertations (Comprehensive Summaries of Uppsala Dissertations from the faculty of Medicine, 1022) - Acta Universitatis Upsalienses, Uppsala, 2001.

15. Pagani MM, Duarte MFS. Profissão Carteiro: qual o nível da flexibiliade nesses profissionais. FIEP Bulletin 2001;70:166.

16. Pagani MM, Duarte MFS. Avaliação do consumo máximo de oxigênio em carteiros convencionais a pé de Florianópolis. FIEP Bulletin 2001;70:167.

17. Hallal PC, Gomez LF, Parra DC, Lobelo F, Mosquera J, Florindo AA, et al. Lições Aprendidas Depois de 10 Anos de Uso do IPAQ no Brasil e Colômbia. J Phys Activ Health 2010;7:S259-S264. 
18. Polito E, Bergamaschi EC. Ginástica laboral: teoria e prática. Rio de Janeiro: Sprint; 2002.

19. Gomes VB, Siqueira KS, Sichieri R. Atividade física em uma amostra probabilística da população do Município do Rio de Janeiro. Cad Saúde Púb 2001;17:969-76.

20. Correios. Concursos. 2010. Disponível em: http:// www.correios.com.br/institucional/concursos/correios/ default.cfm (30 out. 2010)

21. Sena JEA, Pontes LM, Ferreira UMG, Silva JM. Composição corporal e sua relação com o nível de atividade física de taxistas e carteiros de João Pessoa - PB. Fit Perf J 2008;7:20-5.

22. Barros MVG, Nahas MV. Comportamento de risco, auto-avaliação do nível de saúde e percepção de estresse entre trabalhadores da indústria. Rev Saúde Púb 2001;35:554-63.

23. Baretta E, Baretta M, Peres KG. Nível de atividade física e fatores associados em adultos do Município de Joaçaba, SC, Brasil. Cad Saúde Púb 2007;23:1595602.

24. Lima V, Romero J, Ceschini FL. Nível de atividade física de acordo com a participação em programas de ginástica laboral. Rev Bras Ciênc Mov 2006;14:43.
25. Romero J, Lima V, Ceschini FL. Prevalência de dores corporais de acordo com a participação em programa de ginástica laboral. Rev Bras Ciênc Mov 2006;14:103.

26. Sjögaard G, Andersen LL, Vedsted P, Nielsen PK, Jorgensen MB, Olsen HB, et al. Changes in shoulder muscle recruitment, oxygenation, and pain development during repetitive work in workers with myalgia - before and after enrolment in work site physical activity programs. J Biomech 2007;40:337-8.

27. Maciel RH, Albuquerque AMFC, Melzer AC, Leônidas SR. Quem se beneficia dos programas de ginástica laboral? Cad Psicol Soc Trab 2005;8:71-86.

28. Ministério da Saúde. Protocolo de investigação, diagnóstico, tratamento e prevenção de Lesão por Esforços Repetitivos/Distúrbios Osteomoleculares Relacionados ao Trabalho/Secretaria de Políticas de Saúde. Brasília: MS; 2000.

29. WHO. BMI classification. Disponível em: http://www. who.int/bmi/index.jsp?introPage=intro_3.html (16 mar. 2008).

\section{ENDEREÇO PARA CORRESPONDÊNCIA}

Endereço:Laboratório de Biomecânica Campus

Universitário - Trindade - Florianópolis - SC - Brasil CEP: $88040-970$

Telefone: (48) 37218530

E-mails: mariajoanna@matrix.com.br adri@tirloni.com.br diogo.biomecanica@gmail.com moro@cds.ufsc.br 